\title{
miR-371b-5p inhibits endothelial cell apoptosis in monocrotaline-induced pulmonary arterial hypertension via PTEN/PI3K/Akt signaling pathways
}

\author{
GUANGFA ZHU ${ }^{1}$, WENMEI ZHANG ${ }^{1}$, YAN LIU $^{2}$ and SHENGHAO WANG ${ }^{2}$ \\ Departments of ${ }^{1}$ Pulmonary and Critical Care Medicine, and ${ }^{2}$ Infectious Diseases, Beijing Institute of Heart, \\ Lung and Blood Vessel Diseases, Beijing Anzhen Hospital, Capital Medical University, Beijing 100029, P.R. China
}

Received August 19, 2017; Accepted March 16, 2018

DOI: $10.3892 / \mathrm{mmr} .2018 .9614$

\begin{abstract}
Pulmonary arterial hypertension (PAH) is a clinical hemodynamic syndrome. It is characterized by elevated PA pressure and pulmonary vascular resistance. In the present study, the role of microRNA (miRNA/miR)-371b-5p in monocrotaline-induced PAH and the underlying mechanisms were investigated. In a monocrotaline-induced PAH rat model, gene chip and reverse transcription-quantitative polymerase chain reaction were employed to measure miRNA expression levels. The results revealed that $\mathrm{miR}-371 \mathrm{~b}-5 \mathrm{p}$ was downregulated in PAH rats compared with the control group. In addition, in vitro results demonstrated that an miR-371b-5p inhibitor reduced $\mathrm{miR}-371 \mathrm{~b}-5 \mathrm{p}$ expression levels, increased apoptosis and reduced proliferation of pulmonary arterial endothelial cells (PAECs) in rats with monocrotaline-induced $\mathrm{PAH}$. Furthermore, inhibition of miR-371b-5p induced phosphatase and tensin homolog (PTEN) protein expression and suppressed that of phosphoinositide 3-kinase (PI3K) and phosphorylated (p)-Akt in the PAECs. In addition, VO-OHpic, a PTEN inhibitor, reduced the protein expression levels of PTEN in the PAECs and inhibited the effects of anti-miR-371b-5p on cell apoptosis. In addition, LY294002, a PI3K inhibitor, reduced the PI3K and p-Akt protein expression in the PAECs and reversed the effects of miR-371b-5p overexpression on the apoptosis of PAECs in rats with monocrotaline-induced PAH. Collectively, the results of the present study indicate that, in this animal model of PAH, miR-371b-5p inhibits apoptosis of PAECs via PTEN/PI3K/Akt signaling pathways.
\end{abstract}

Correspondence to: Dr Guangfa Zhu, Department of Pulmonary and Critical Care Medicine, Beijing Institute of Heart, Lung and Blood Vessel Diseases, Beijing Anzhen Hospital, Capital Medical University, 2 Anzhen Road, Chaoyang, Beijing 100029, P.R. China

E-mail: guangfa_zhu@ccmu.edu.cn

Key words: miR-371b-5p, pulmonary arterial hypertension, monocrotaline, PTEN, PI3K, Akt

\section{Introduction}

Pulmonary arterial hypertension (PAH) is a clinical hemodynamic syndrome. It is characterized by elevated pulmonary artery (PA) pressure and pulmonary vascular resistance (1). This may lead to right cardiac failure and mortality (1). A mean PA pressure $\geq 25 \mathrm{mmHg}$ under rest state and $\geq 30 \mathrm{mmHg}$ under motion state are the diagnostic criteria of PAH. PAH is associated with high morbidity (2). According to epidemiological data in the USA, the mortality rate of all types of PAH is $>30-50 / 1$ million. At present, treatment for PAH is limited. Thus, it is associated with high mortality and markedly poor prognosis (2). In addition, other heart diseases may be associated with PAH at moderate and advanced stages of the condition. This leads to notably poor prognosis, and high morbidity, disability rate and mortality (1). Medically developed countries have made notable advances in fields of PAH basic research, diagnostic technology and treatment (3); however, these fields in China have not been extensively studied, thus domestic developments are difficult to conduct in such fields (3).

MicroRNAs (miRNAs/miRs) are a class of endogenous noncoding small RNA molecules 21 nucleotides in length (4), which bind with the 3 ' untranslated region (UTR) of mRNA. Thus, they affect the stability of mRNA or inhibit protein translation. As a result, miRNAs negatively regulate target gene expression at the post-transcriptional level (4). It is estimated that miRNAs participate in the regulation of $>30 \%$ of established human genes and regulate almost all pathophysiological processes of the body (4). Alterations in miRNA expression under hypoxic conditions have been reported to participate in PAH formation (5). In addition, miRNAs have been demonstrated to regulate the proliferation, apoptosis and migration of pulmonary vascular smooth muscle cells (5).

Phosphatase and tensin homolog (PTEN) was the first tumor suppressor gene with dual-phosphatase activity and was identified in research into primary breast cancer in 1997 (6). PTEN is involved in numerous physiological and pathological processes of the body (6) and is extensively distributed in normal tissue. In addition, it promotes cell apoptosis and reduces cell proliferation under normal physiological conditions (7). PTEN also has important effects on tumor cell growth, proliferation, differentiation, apoptosis and 
migration under pathological states (8). It has been reported that PTEN protein phosphorylation may improve the stability of PTEN (7). Furthermore, the PTEN phosphatase catalysis region can be inhibited rendering loss of its activity (8). PTEN and phosphorylated (p)-PTEN exhibit opposing effects in the regulation of cell proliferation and apoptosis (6).

Phosphoinositide 3-kinase (PI3K)/Akt-endothelial nitric oxide synthase (eNOS)-mediated NO production has been detected in coronary artery, aorta, mesentery, kidney and iliac artery endothelial cells (9). Increasing evidence indicates that the PI3K/Akt signaling pathway is involved in vasoconstriction and remodeling (10), and this signaling pathway is an important target of vasofunctional drugs (10). A vascular ring model in vitro revealed that a vasoactive intestinal peptide relaxed the mouse pulmonary arterial ring, which may occur through the activation of the pulmonary vascular endothelial cell PI3K/Akt signaling pathway (11). Additionally, another study reported that estrogen may activate the PI3K/Akt signaling pathway. In this manner, PI3K/Akt can regulate the bioactivity of pulmonary vascular system (11). These studies have indicated that regulating the PI3K/Akt-eNOS signaling pathway is of great importance in the treatment of PAH $(9,11)$. In the present study, the function of miR-371b-5p in monocrotaline-induced $\mathrm{PAH}$ and the underlying mechanisms were investigated.

\section{Materials and methods}

Animals andPAHratmodel.A total of 12 male Sprague-Dawley rats (200-230 g, 6-8 weeks, n=12) were purchased from Beijing Vital River Laboratory Animal Technology Co., Ltd. (Beijing, China) for all treatments and housed at $22-23^{\circ} \mathrm{C}$, $55-60 \%$ humidity, 12-h light/dark cycle and freely available food and water. All animal care and experimental procedures were performed with the approval of the Institutional Animal Care and Use Committee of Capital Medical University (Beijing China). The present study was approved by the ethics committee of Beijing Anzhen Hospital (Beijing, China). All rats were randomly distributed into two groups: Control $(n=6)$ and PAH model groups $(n=6)$. In the PAH model group, rats were induced with $60 \mathrm{mg} / \mathrm{kg} /$ three days monocrotaline (intraperitoneal injection; Sigma-Aldrich; Merck KGaA, Darmstadt, Germany) and exposed to normobaric hypoxia conditions $\left(10 \% \mathrm{pO}_{2}\right)$ with an automatic oxygen controller (ProOx Model 110; Biospherix, Ltd., Parish, NY, USA) for 21 days. The control mice were treated with normal saline for 21 days.

Histological findings of PAH. After 21 days of induction, mice were anesthetized using $35 \mathrm{mg} / \mathrm{kg}$ pentobarbital sodium and sacrificed by decollation. Lung tissues ( $n=3 /$ every group) were washed with PBS and fixed with $10 \%$ buffered formalin for $24 \mathrm{~h}$ at room temperature. Subsequently, lung tissues were embedded in paraffin and cut into $4-\mu \mathrm{m}$ sections. Sections were stained with hematoxylin and eosin (H\&E) for $5 \mathrm{~min}$ at room temperature.

Cell culture and transfection. PA tissue samples of PAH rats $(n=3)$ were incubated in Hanks' solution containing collagenase $(1.5 \mathrm{mg} / \mathrm{ml}$; Invitrogen; Thermo Fisher Scientific, Inc., Waltham, MA, USA) for $30 \mathrm{~min}$ at $4^{\circ} \mathrm{C}$. Adventitia was carefully stripped off with a fine forcep and the endothelium was removed. The remaining smooth muscle was digested with collagenase and elastase for $50 \mathrm{~min}$ at $37^{\circ} \mathrm{C}$. The pulmonary arterial endothelial cells (PAECs) were collected and cultured in Dulbecco's modified Eagle's medium (Gibco; Thermo Fisher Scientific, Inc.), $10 \%$ fetal bovine serum (Gibco; Thermo Fisher Scientific, Inc.), 50 units $/ \mathrm{ml}$ penicillin, and $50 \mu \mathrm{g} / \mathrm{ml}$ streptomycin at $37^{\circ} \mathrm{C}$ in a $5 \% \mathrm{CO}_{2}$ incubator. Following culture to $70-80 \%$ confluence, cells were transfected with $100 \mathrm{ng}$ miR-371b-5p (5'-aagugccccacaguuugagugc-3'), $100 \mathrm{ng}$ anti-miR-371b-5p (5'-ggtaacactcaaaagatggc-3') or $100 \mathrm{ng}$ negative control (NC) miRNA (used for both anti-NC/miR-NC; 5'-CCCCCCCCC CCCC-3') using Lipofectamine 2000 (Invitrogen; Thermo Fisher Scientific, Inc.). These mimics were purchased from Sangon Biotech (Shanghai) Co., Ltd. (Shanghai, China). A total of $20 \mathrm{nM}$ of VO-OHpic (MedChemExpress USA, Monmouth Junction, NJ, USA), a PTEN inhibitor was added into cell after transfection for $4 \mathrm{~h}$ and incubated for $48 \mathrm{~h}$.

Reverse transcription-quantitative polymerase chain reaction $(R T-q P C R)$ and gene microarray hybridization. Total miRNA was extracted from lung tissue samples or transfected PAECs with a NucleoSpin miRNA isolation kit (Takara Bio, Inc., Otsu, Japan). Total RNA (200 ng) was reverse transcribed to cDNA using an PrimeScript ${ }^{\mathrm{TM}}$ RT reagent kit with gDNA Eraser (Takara Biotechnology Co., Ltd., Dalian, China) at $37^{\circ} \mathrm{C}$ for $15 \mathrm{~min}$ and at $85^{\circ} \mathrm{C}$ for $5 \mathrm{sec}$. qPCR was performed with amiScript SYBR ${ }^{\circledR}$ Green PCR kit (Qiagen GmbH, Hilden, Germany) using a Rotor Gene 6000 Real-Time PCR Machine (Qiagen $\mathrm{GmbH})$. Sequence of miR-371b-5p forward: 5'-gtggcactcaaa ctgt-3' and reverse: 5'-catcttttgagtgttac-3'; U6 forward: 5'-CAA ATTCGTGAAGCGTT-3'; reverse: 5'-TGGTGTCGTGGAGTC G-3. RT-qPCR were amplified by PCR in the following conditions: Pre-denaturation for $15 \mathrm{~min}$ at $94^{\circ} \mathrm{C}$; 40 cycles of $30 \mathrm{sec}$ at $94^{\circ} \mathrm{C}, 40 \mathrm{sec}$ at $55^{\circ} \mathrm{C}, 1 \mathrm{~min}$ at $72^{\circ} \mathrm{C}$ and extension for $5 \mathrm{~min}$ at $72^{\circ} \mathrm{C}$. Gene expression was analyzed using $2^{-\Delta \Delta \mathrm{Cq}}$ method (12).

For microarray hybridization, total RNA obtained from the PAH model and control rat lung tissues (500 ng) was employed and labeled with cyanine-3-cytidine triphosphate (Agilent Technologies, Inc., Santa Clara, CA, USA) at $60^{\circ} \mathrm{C}$ for $30 \mathrm{~min}$, and subsequently hybridized to the Agilent Mouse miRNA microarray (KG4471A-021828 platform) on the Axon GenePix ${ }^{\circledR}$ 4000B microarray scanner (Agilent Technologies, Inc., Santa Clara, CA, USA). Images were quantified and feature-extracted using Agilent Feature Extraction software version 10.7.3.1 (Agilent Technologies, Inc.).

MTT assay. Following PAEC transfection for 24, 48 and $72 \mathrm{~h}$, $15 \mu \mathrm{l}$ MTT (5 mg/ml; Promega Corporation, Madison, WI, USA) was added to PA cells $\left(1 \times 10^{3}\right.$ cell/well) for $4 \mathrm{~h}$ with $5 \%$ $\mathrm{CO}_{2}$ at $37^{\circ} \mathrm{C}$. Old medium was removed and $150 \mu \mathrm{l}$ dimethyl sulfoxide was added to cells for $20 \mathrm{~min}$ at $37^{\circ} \mathrm{C}$. A microplate reader (SpectraMax M5; Molecular Devices, LLC, Sunnyvale, CA, USA) was employed and absorbance was measured at $490 \mathrm{~nm}$.

Flow cytometry for apoptosis. Following PAEC transfection for $48 \mathrm{~h}$, cells were washed with $1 \mathrm{ml} /$ well PBS for three times and resuspended with buffer (cat. no. 556420; BD Biosciences,

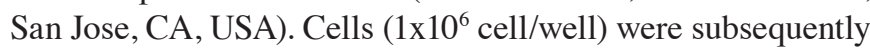
stained with $10 \mu \mathrm{l}$ annexinV-fluorescein isothiocyanate 


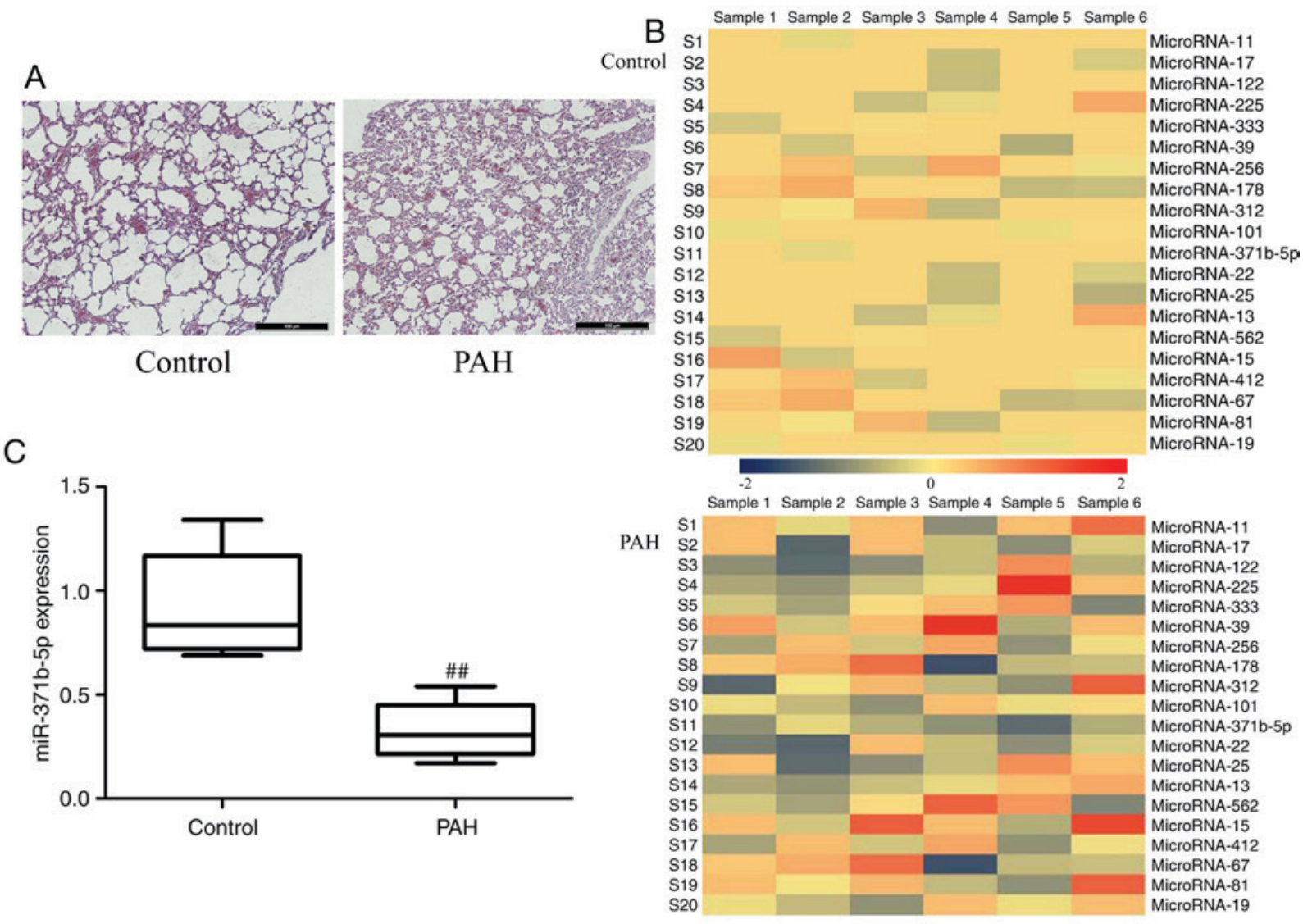

Figure 1. Expression of miR-371b-5p in the lung tissues of monocrotaline-induced PAH rat models. (A) Hematoxylin and eosin staining for lung tissues (magnification, x100). (B) Microarray analysis of microRNA expression in the lung tissues from PAH model and control rats. (C) Reverse transcription-quantitative polymerase chain reaction analysis of miR-371b-5p expression in the lung tissues from PAH model and control rats. ${ }^{\# \#} \mathrm{P}<0.01 \mathrm{vs}$. control group. miR, microRNA; PAH, pulmonary arterial hypertension; S, sample.

$(20 \mu \mathrm{g} / \mathrm{ml})$ and $5 \mu \mathrm{l}$ propidium iodide $(50 \mu \mathrm{g} / \mathrm{ml})$ staining solution (cat. no. 556420; BD Biosciences) for 15 min in darkness at room temperature. The rate of apoptosis was analyzed by flow cytometry (c6; BD Biosciences) and using Flowjo 7.6.1 (FlowJo, LLC, Ashland, OR, USA).

Commercial enzyme activity kits. LDH activity levels were measured using LDH activity kits (cat. no. C0017; Beyotime Institute of Biotechnology). A microplate reader (SpectraMax M5; Molecular Devices, LLC, Sunnyvale, CA, USA) was employed and absorbance was measured at 490 nm. Caspase-3/9 activity was measured using Caspase-3/9 activity kits (cat. nos. C1115 and C1157; Beyotime Institute of Biotechnology). A microplate reader (SpectraMax M5; Molecular Devices, LLC, Sunnyvale, CA, USA) was employed and absorbance was measured at $405 \mathrm{~nm}$.

miR-371b-5p target reporter assay. In the present study, a dual-luciferase miR-371b-5p target reporter vector was generated, which consisted of a double-stranded oligonucleotide containing the 3'UTR of PTEN using http://www.targetscan. org. The PTEN 3'UTR was cloned into Pmel and XbaI sites in pmirGLO Dual-Luciferase miRNA Target Expression vectors (Promega Corporation). Sequence of miR-371b-5p forward: 5'-cagacatgacagccatcatcaaa-3' and 5'-aagagggataaaacacca-3'; PTEN plasmid (5'-TCCTGGAGCGGGGGGGAG AA-3' and 5'-GTATATAATAAGTATAATAT-3'). A total of
$100 \mathrm{ng}$ of PTEN plasmid and $100 \mathrm{ng}$ of miR-371b-5p mimics were transfected using Lipofectamine 2000 (Invitrogen; Thermo Fisher Scientific, Inc.). Following PAEC transfection $\left(1 \times 10^{5} \mathrm{cell} / \mathrm{ml}\right)$ for $48 \mathrm{~h}$, the luciferase activity was measured using the Dual-Luciferase Reporter Assay system (Promega Corporation) and the firefly luciferase construct was normalized to Renilla luciferase.

Western blot analysis. Following PAEC transfection for $48 \mathrm{~h}$, cells were collected and washed with PBS three times. Cell protein was extracted using radioimmunoprecipitation assay lysis buffer (Beyotime Institute of Biotechnology) and total protein was quantified via a bicinchoninic acid protein kit (BCA; Beyotime Institute of Biotechnology). Total protein (30-80 $\mu \mathrm{g}$ ) was separated by 6-12\% SDS-PAGE and was transferred onto polyvinylidenedifluoride (PVDF) membranes. The PVDF membranes were blocked with $5 \%$ non-fat milk in TBST for $1 \mathrm{~h}$ at $37^{\circ} \mathrm{C}$ and incubated overnight with antibodies against eNOS (cat. no. sc-49055; 1:500; Santa Cruz Biotechnology, Inc.), AP-1 (cat. no. ab21981; 1:1,000; Abcam) and KLF-2 (cat. no. ab203591; 1:1,000; Abcam), PTEN (cat. no. sc-9145; 1:1,000; Santa Cruz Biotechnology, Inc.), PI3K (cat. no. sc-7174; 1:1,000; Santa Cruz Biotechnology, Inc.), phosphorylated (p)-Akt (cat. no. sc-7985-R; 1:500; Santa Cruz Biotechnology, Inc.) and GAPDH (cat. no. sc-25778; 1:5,000; Santa Cruz Biotechnology, Inc.) at $4^{\circ} \mathrm{C}$. The PVDF membranes were washed with TBST three times and incubated 
A
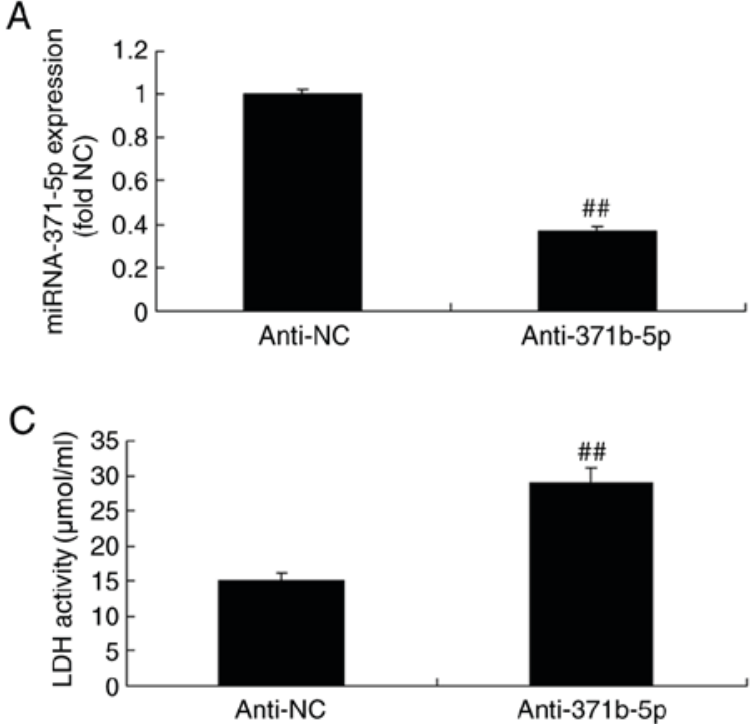

$\mathrm{E}$

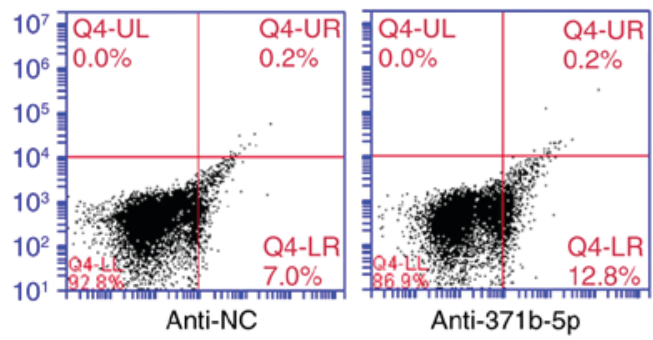

B

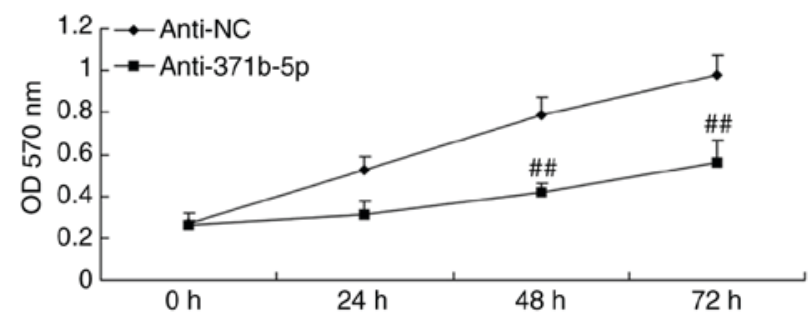

$\mathrm{D}$
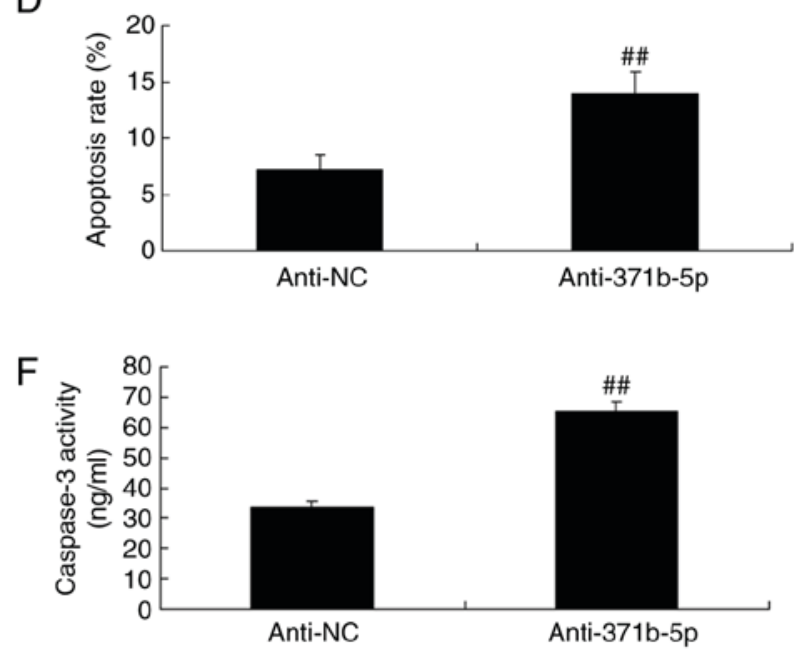

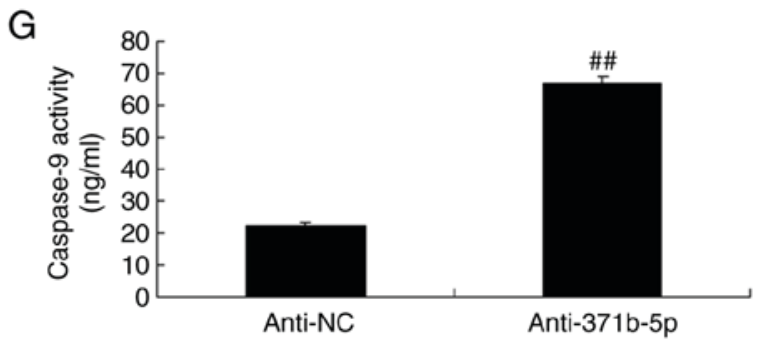

Figure 2. Downregulation of miR-371b-5p increases pulmonary arterial endothelial cell death from rats with monocrotaline-induced pulmonary arterial hypertension. (A) miR-371b-5p expression was determined by reverse transcription-quantitative polymerase chain reaction. (B) Cell proliferation was measured by an MTT assay. (C) LDH activity was measured in cells transfected with anti-miR-371b-5p or anti-NC. (D) Cell apoptosis was quantified by flow cytometry following annexin V-fluorescein isothiocyanate/propidium iodide staining (E) Q4-UR and Q4-LR indicate apoptotic cells). Representative flow cytometry plots following staining for apoptosis. The activity of (F) caspase-3 and (G) caspase-9 was also measured in cells transfected with anti-miR-371b-5p or anti-NC. ${ }^{\# \prime} \mathrm{P}<0.01$ vs. anti-NC group. miR/miRNA, microRNA; LDH, lactate dehydrogenase; NC, negative control; anti-371b-5p, anti-miR-371b-5p; OD, optical density.

with goat anti-rabbit IgG-HRP (cat. no. sc-2004; 1:5,000; Santa Cruz Biotechnology, Inc.) for $1 \mathrm{~h}$ at $37^{\circ} \mathrm{C}$. Protein bands were visualized via by BeyoECL Plus (Beyotime Institute of Biotechnology) and analyzed using sodium Image Lab 3.0 (Bio-Rad Laboratories, Inc.).

Immunofluorescence analysis. Following PAEC transfection for $48 \mathrm{~h}$, cells $\left(1 \times 10^{5}\right.$ cell/well) were washed with PBS twice and fixed with $4 \%$ paraformaldehyde for $15 \mathrm{~min}$ at room temperature. Cells were subsequently incubated with Triton X-100 (Sigma-Aldrich; Merck KGaA) for $15 \mathrm{~min}$ at room temperature, blocked with $5 \% \mathrm{BSA}$ in PBS for $1 \mathrm{~h}$ at $37^{\circ} \mathrm{C}$ and further incubated with PTEN (cat. no. sc-9145; 1:100; Santa Cruz Biotechnology, Inc.) antibody at $4{ }^{\circ} \mathrm{C}$ overnight. Cells were visualized with donkey anti-rabbit IgG-CFL 555 (1:1,000; cat. no. sc-362271; Santa Cruz Biotechnology, Inc.) for $1 \mathrm{~h}$ at room temperature. Cells were subsequently stained with DAPI for $30 \mathrm{~min}$ at room temperature and observed using x200 magnification (T300 confocal microscope, Nikon Corporation, Tokyo, Japan).

Statistical analysis. Data are presented as the mean \pm standard deviation using SPSS 17.0 (SPSS, Inc., Chicago, IL, USA). Data were analyzed using a Student's t-test or one-way analysis of variance by Tukey's post hoc test. $\mathrm{P}<0.05$ was considered to indicate a statistically significant difference.

\section{Results}

Expression of miR-371b-5p in monocrotaline-induced PAH rats. H\&E staining revealed that lung tissue was damaged and PAEC apoptosis was observed in monocrotaline-induced $\mathrm{PAH}$ 
A

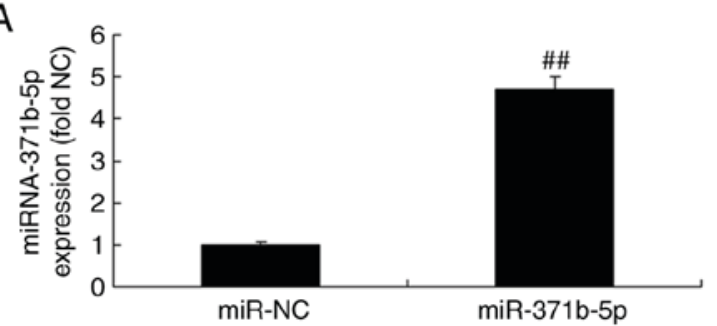

C

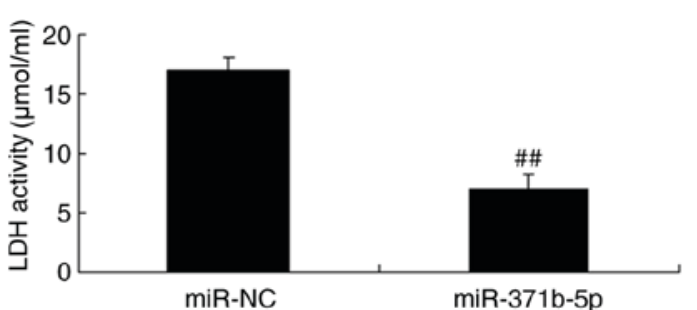

E
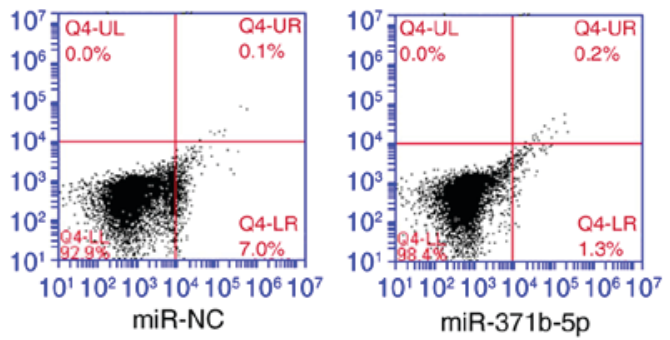

B

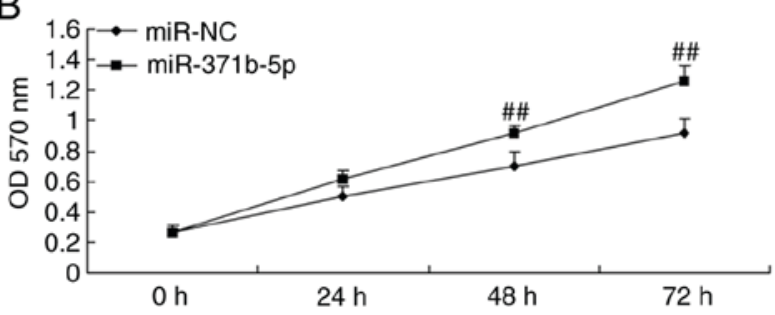

D

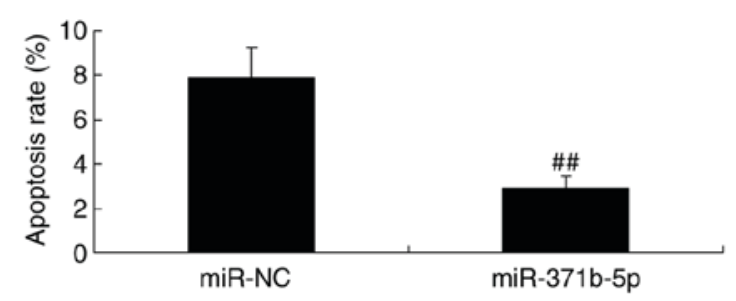

$\mathrm{F}$

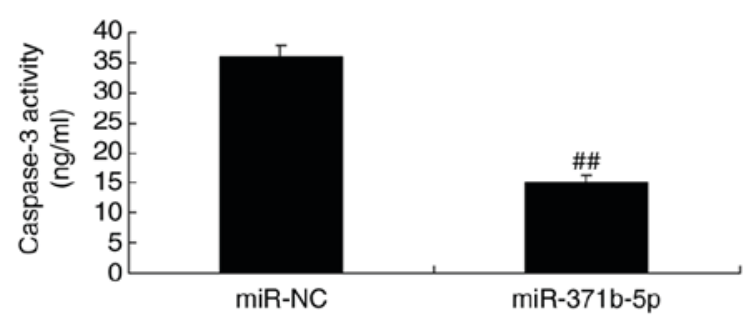

G

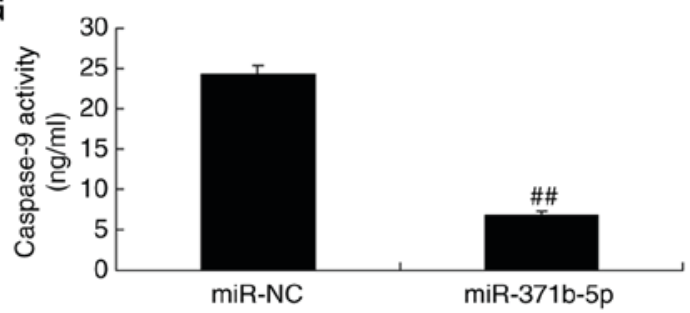

Figure 3. Upregulation of miR-371b-5p decreases pulmonary arterial endothelial cell death from rats with monocrotaline-induced pulmonary arterial hypertension. (A) miR-371b-5p expression was determined by reverse transcription-quantitative polymerase chain reaction. (B) Cell proliferation was measured by an MTT assay. (C) LDH activity was measured in cells transfected with miR-371b-5p or miR-NC. (D) Cell apoptosis was quantified by flow cytometry following annexin V-fluorescein isothiocyanate/propidium iodide staining (E) Q4-UR and Q4-LR indicate apoptotic cells). Representative flow cytometry plots following staining for apoptosis. The activity of (F) caspase-3 and $(\mathrm{G})$ caspase-9 was also measured in cells transfected with miR-371b-5p or miR-NC. ${ }^{\# \#} \mathrm{P}<0.01$ vs. miR-NC group. miR/miRNA, microRNA; LDH, lactate dehydrogenase; NC, negative control; OD, optical density.

rats, compared with the control group (Fig. 1A). The results of microarray analysis demonstrated that miR-371b-5p expression levels were downregulated in monocrotaline-induced PAH rats compared with in the control group (Fig. 1B). Subsequently, RT-qPCR analysis confirmed that miR-371b-5p was significantly downregulated in the monocrotaline-induced lung tissue of PAH group compared with in the control group (Fig. 1C). These results indicate that miR-371b-5p may be involved in monocrotaline-induced $\mathrm{PAH}$, and the underlying mechanisms require investigation.

Downregulation of miR-371b-5p increases PAEC death from rats with monocrotaline-induced $P A H$. In the in vitro study, RT-qPCR analysis confirmed that the expression levels of miR-371b-5p were significantly reduced in an in vitro model of PAH following cell transfection with anti-miR-371b-5p, compared with the $\mathrm{NC}$ group (Fig. 2A). In addition, the results revealed that the suppression of miR-371b-5p expression significantly inhibited cell proliferation, increased lactate dehydrogenase (LDH) activity and induced cell apoptosis in the anti-miR-371b-5p group, compared with the NC group (Fig. 2B-E). Caspase-3/9 activities were also significantly promoted by the inhibition of miR-371b-5p expression in the anti-miR-371b-5p group, compared with the NC group (Fig. 2F and $\mathrm{G})$.

Upregulation of $m i R-371 b-5 p$ decreases $P A E C$ proliferation from rats with monocrotaline-induced $P A H$. The expression of miR-371b-5p was induced in the in vitro study using miR-371b-5p mimics to analyze the function of miR-371b-5p in PAECs. As presented in Fig. 3A, there was a significant increase in miR-371b-5p expression in cells transfected with miR-371b-5p mimics compared with the NC group. Furthermore, upregulation of miR-371b-5p significantly 
A
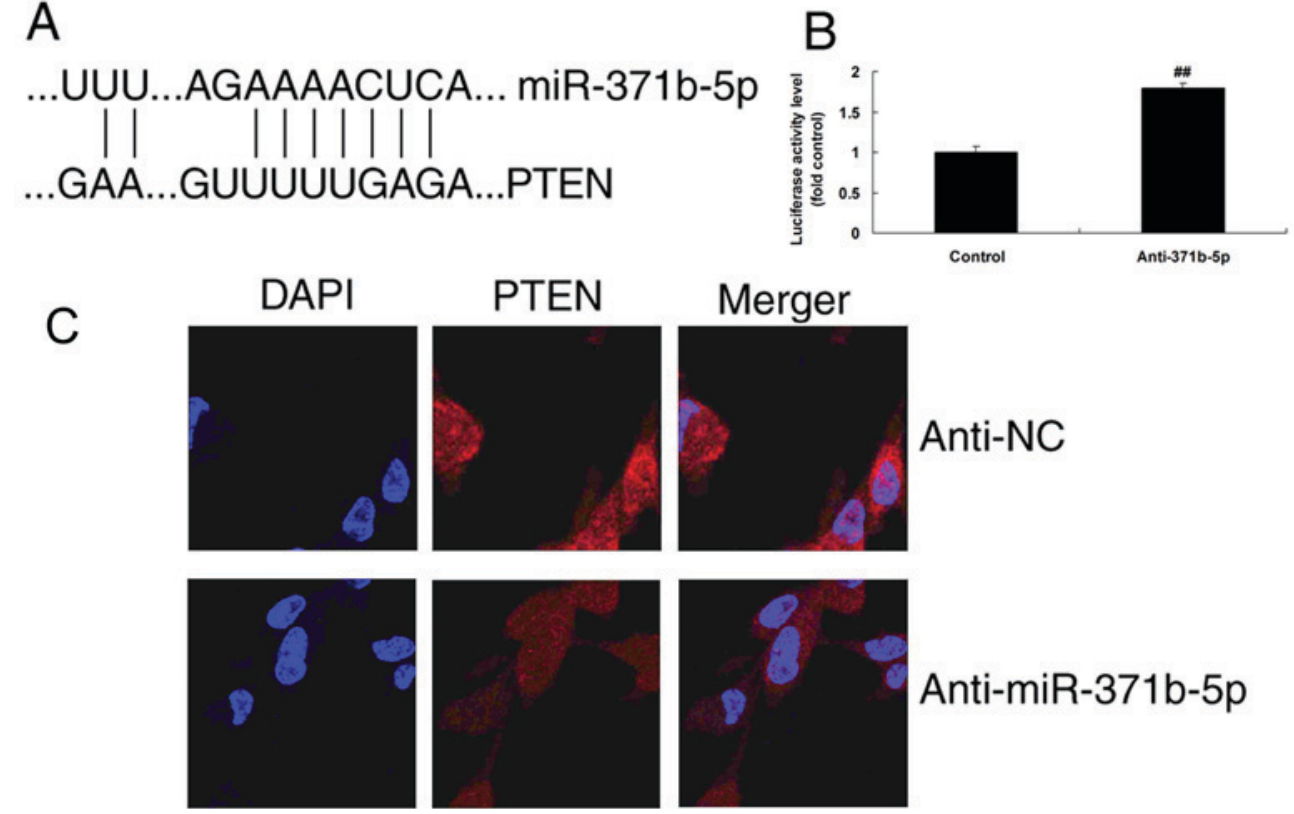

Anti-miR-371b-5p

Figure 4. Effects of anti-miR-371b-5p on PTEN signaling in pulmonary arterial endothelial cells from rats with monocrotaline-induced pulmonary arterial hypertension. (A) Predicted miR-371b-5p target site in the 3' untranslated region of PTEN. (B) Luciferase activity, (C) PTEN protein expression was detected by immunofluorescence staining (magnification, $\mathrm{x} 100$ ). ${ }^{\sharp \# \mathrm{P}} \mathrm{P}<0.01$ vs. control group. miR, microRNA; PTEN, phosphatase and tensin homolog; NC, negative control.

promoted cell proliferation, and inhibited LDH activity and PAEC apoptosis compared with the NC group (Fig. 3B-E). In addition, the upregulation of miR-371b-5p inhibited caspase-3/9 activity in the miR-371b-5p group compared with the NC group (Fig. 3F and G).

Effects of miR-371b-5p on the PAECs may occur via PTEN/PI3K/Akt signaling pathways in vitro. To investigate the mechanism by which miR-371b-5p functions in $\mathrm{PAH}$, alterations in PTEN/PI3K/Akt signaling pathways were analyzed in PAECs following miR-371b-5p transfection. The predicted binding site of miR-371b-5p in the 3'UTR of PTEN is presented in Fig. 4A. Then, luciferase activity levels were increased by downregulation of miR-371b-5p, compared with the control group (Fig. 4B). In addition, immunofluorescence analysis demonstrated that the upregulation of miR-371b-5p induced PTEN protein expression in the anti-miR-371b-5p group, compared with the NC group (Fig 4C).

Furthermore, as presented in Fig. 5A-D, western blot analysis demonstrated that the downregulation of miR-371b-5p significantly induced PTEN protein expression levels and significantly suppressed the protein expression levels of PI3K and p-Akt in the anti-miR-371b-5p group, compared with the NC group. However, upregulation of miR-371b-5p significantly suppressed PTEN protein expression and significantly induced the expression of PI3K and $\mathrm{p}-\mathrm{Akt}$ protein in the anti-miR-371b-5p group, compared with the NC group (Fig. 5E-H). These results indicated that miR-371b-5p/PTEN/PI3K/Akt signaling pathways may be associated with PAH.

Inhibition of PTEN reduces the effects of anti-miR-371b-5p on PAEC death in vitro. To further confirm the function of PTEN in the effects of anti-miR-371b-5p on PAEC death, a PTEN inhibitor was employed to reduce PTEN expression following anti-miR-371b-5p transfection. As demonstrated in Fig. 6A and B, PTEN inhibitor significantly reduced PTEN expression in PAECs with PTEN inhibitor treatment following anti-miR-371b-5p transfection, compared with the anti-miR-371b-5p-transfected group without PTEN inhibitor treatment. Additionally, the inhibition of PTEN significantly increased the protein expression levels of PI3K and p-Akt in PAECs following anti-miR-371b-5p transfection, compared with the anti-miR-371b-5p-transfected group without PTEN inhibitor treatment (Fig. 6A, C and D). Furthermore, the inhibition of PTEN significantly promoted cell proliferation, and inhibited LDH activity and apoptosis of PAECs following anti-miR-371b-5p-transfection, compared with the anti-miR-371b-5p-transfected group without PTEN inhibitor treatment (Fig. 6E-H). The effect of the PTEN inhibitor on apoptosis in anti-miR-371b-5p-transfected cells was confirmed by the results of the caspase-3/9 activities, which were reduced compared with the anti-miR-371b-5p-transfected group without PTEN inhibitor treatment (Fig. 6I and J).

Inhibition of PI3K increases the effects of miR-371b-5p on $P A E C$ death in vitro. The role of PI3K/Akt signaling in the effects of miR-371b-5p on PAEC death was investigated in the present study. As presented in Fig. 7A-C, treatment with a PI3K inhibitor significantly suppressed p-Akt and PI3K protein expression levels in PAECs following miR-371b-5p transfection, compared with miR-371b-5p-transfected cells without PI3K inhibitor treatment. In addition, the inhibition of PI3K reduced cell proliferation, and induced LDH activity and apoptosis of PAECs following miR-371b-5p transfection, compared with miR-371b-5p-transfected group without PI3K inhibitor treatment (Fig. 7D-G). The effect of the PI3K inhibitor on apoptosis in miR-371b-5p-transfected cells was confirmed by the results of the caspase-3/9 activities, which were increased compared with the miR-371b-5p-transfected 
A

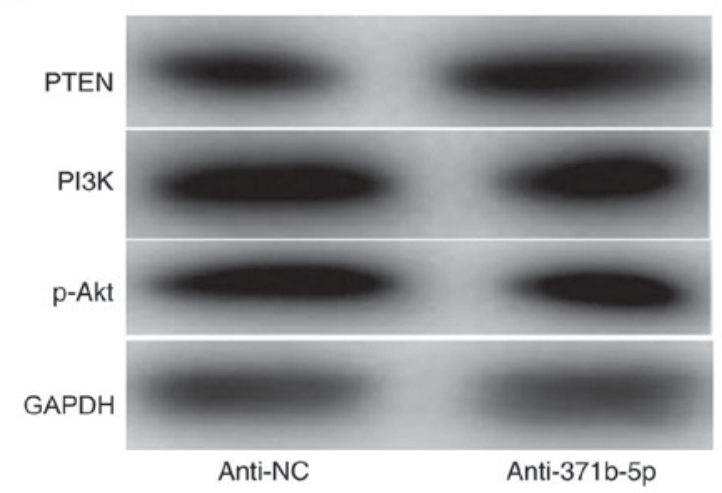

C

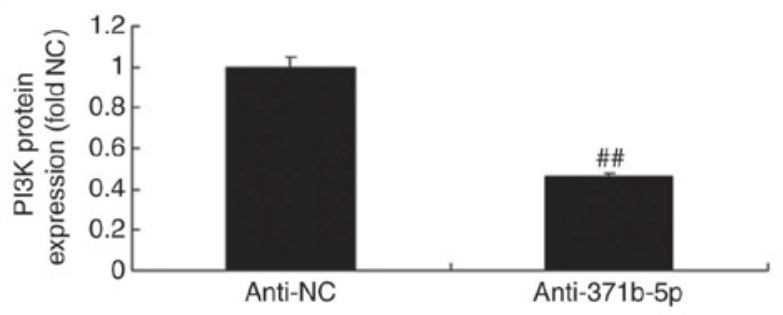

E

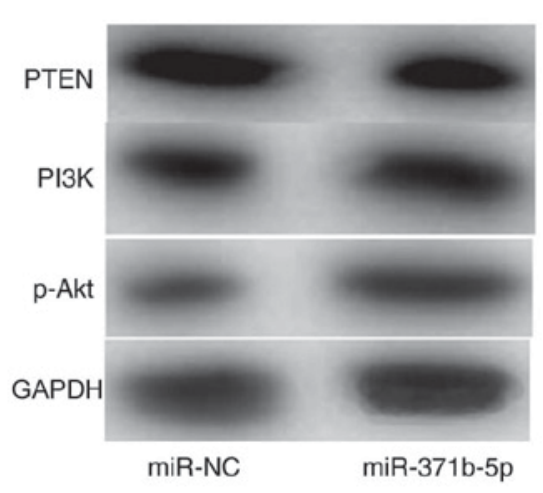

G

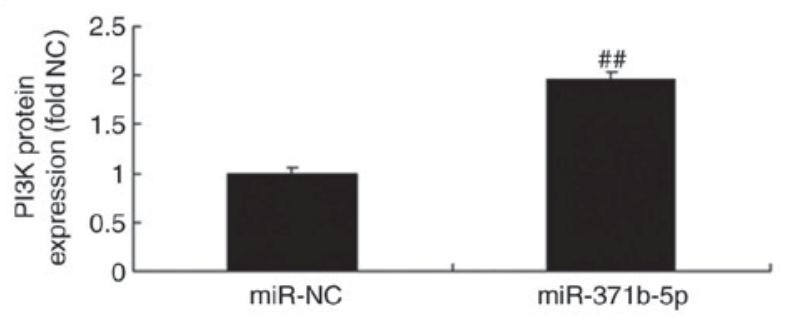

B

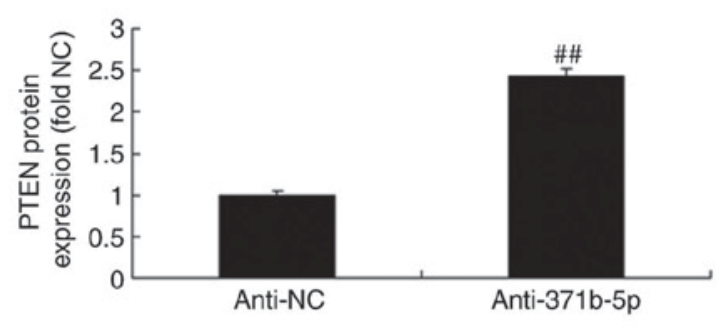

$\mathrm{D}$

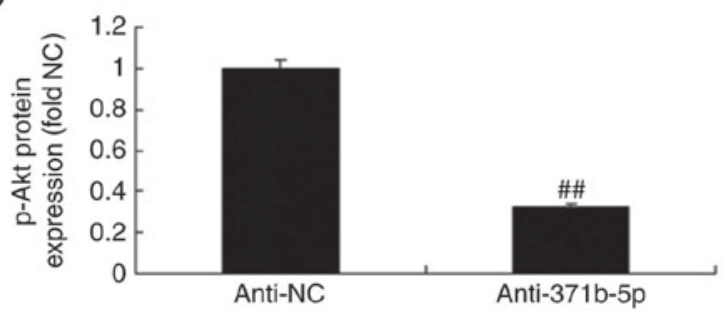

F

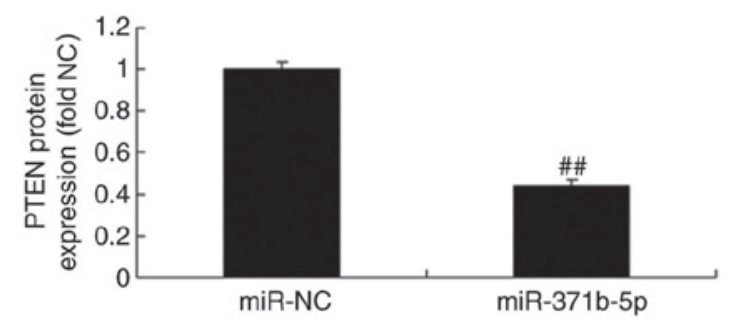

$\mathrm{H}$

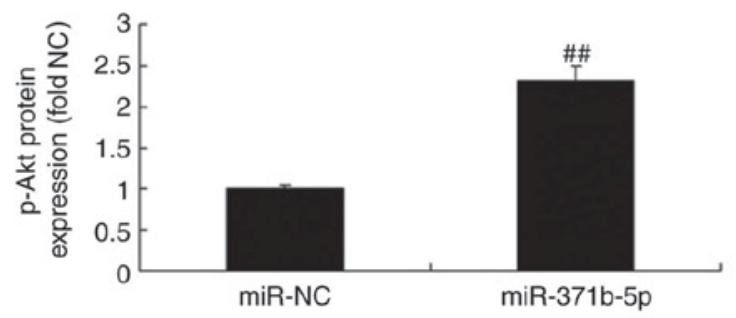

Figure 5. Effects of miR-371b-5p on PTEN/PI3K/Akt signaling pathways in pulmonary arterial endothelial cells from rats with monocrotaline-induced pulmonary arterial hypertension. (A) Representative western blot bands for the protein expression of PTEN, PI3K and p-Akt following transfection of cells with anti-miR-371b-5p or anti-NC. Densitometric analysis was performed to quantify and statistically analyze the protein expression of (B) PTEN, (C) PI3K and (D) p-Akt. (E) Representative western blot bands for the protein expression of PTEN, PI3K and p-Akt following transfection of cells with miR-371b-5p or miR-NC. Densitometric analysis was performed to quantify and statistically analyze the protein expression of (F) PTEN, (G) PI3K and (H) p-Akt. ${ }^{\# \# P<0.01 ~}$ vs. anti-NC or miR-NC group. miR, microRNA; PTEN, phosphatase and tensin homolog; PI3K, phosphoinositide 3-kinase; p-, phosphorylated-; NC, negative control; anti-371b-5p, anti-miR-371b-5p.

group without PI3K inhibitor treatment (Fig. 7H and I). These results indicated that miR-371b-5p may regulate PAEC death via PI3K/Akt signaling pathway by PTEN expression; however, the downstream channel of PTEN/PI3K/Akt signaling pathways has a great number of roles in PAH and requires further investigation.
Effects of miR-371b-5p on eNOS, AP-1 and KLF-2 levels in PAECs. The effects of miR-371b-5p on downstream PTEN/PI3K/Akt signaling pathways were investigated in PAECs; eNOS, AP-1 and KLF-2 expression levels were analyzed by western blotting. As presented in Fig. 8A-D, anti-miR-371b-5p significantly suppressed eNOS protein 

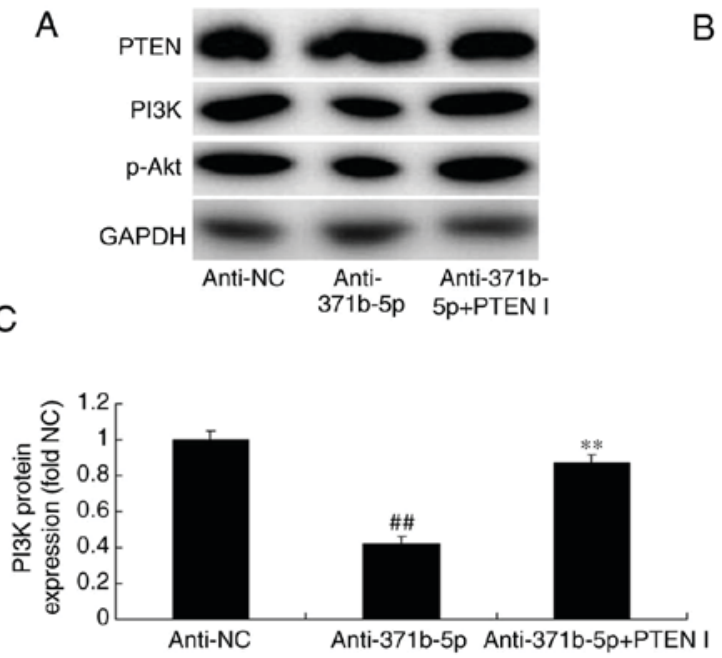

\section{$\mathrm{E}$}

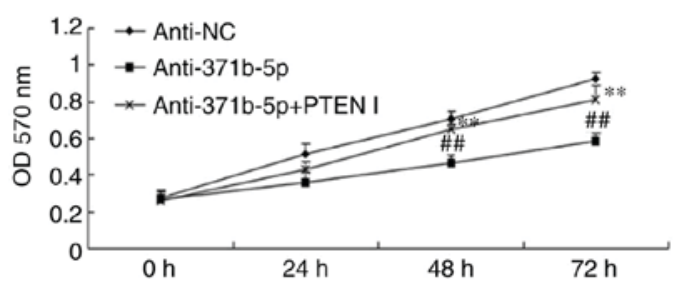

G

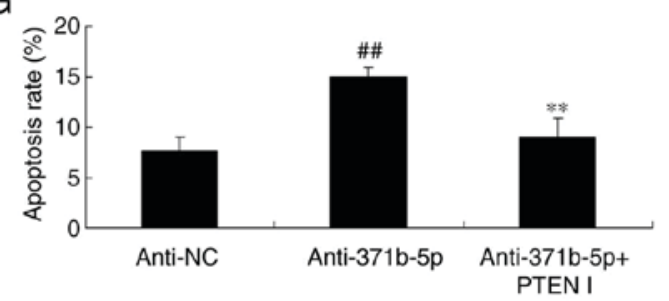

I

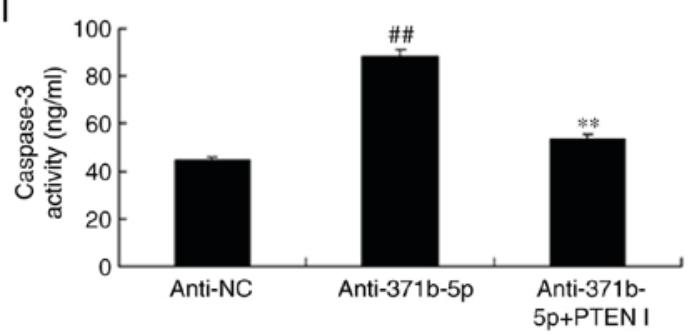

B

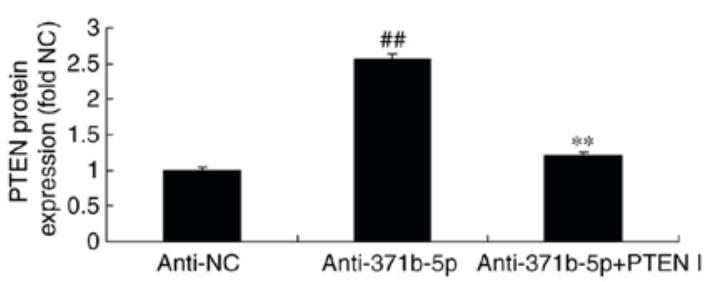

D

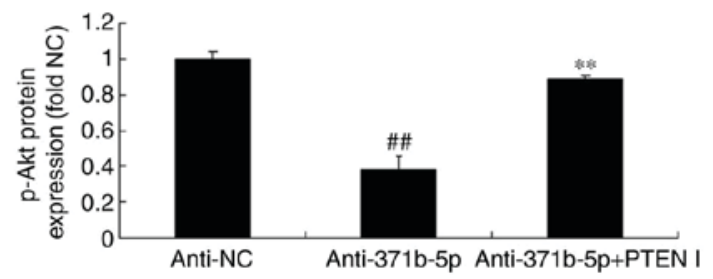

$\mathrm{F}$

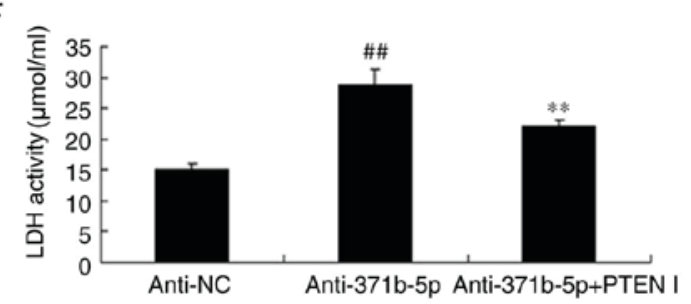

$\mathrm{H}$

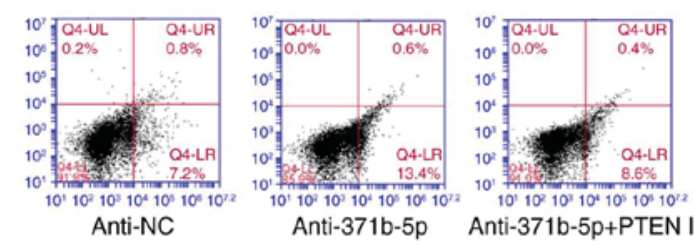

$J$

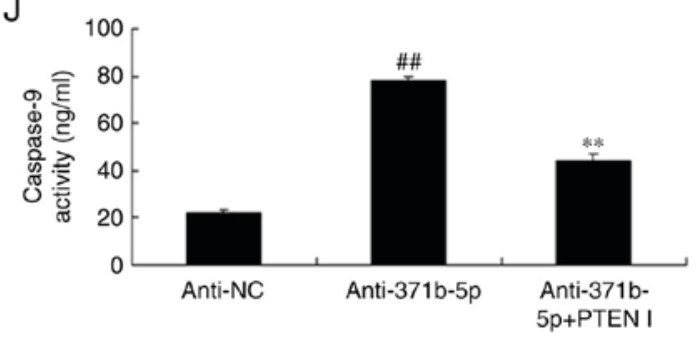

Figure 6. Inhibition of PTEN reduces the effects of anti-miR-371b-5p on lung cell death in an in vitro model of pulmonary artery hypertension. (A) Representative western blot bands for the protein expression of PTEN, PI3K and p-Akt following transfection of cells with anti-miR-371b-5p, with or without PTEN inhibitor treatment, or anti-NC. Densitometric analysis was performed to quantify and statistically analyze the protein expression of (B) PTEN, (C) PI3K and (D) p-Akt. (E) Cell proliferation was measured by an MTT assay. (F) LDH activity was measured in cells transfected with anti-miR-371b-5p, with or without PTEN inhibitor treatment, or anti-NC. (G) Cell apoptosis was quantified by flow cytometry following annexin V-fluorescein isothiocyanate/propidium iodide staining (H) Q4-UR and Q4-LR indicate apoptotic cells). Representative flow cytometry plots following staining for apoptosis. The activity of (I) caspase-3 and (J) caspase-9 was also measured in cells transfected with anti-miR-371b-5p, with or without PTEN inhibitor treatment, or anti-NC. ${ }^{\#} \mathrm{P}<0.01 \mathrm{vs}$. anti-NC group; ${ }^{* *} \mathrm{P}<0.01$ vs. anti-371b-5p group. PTEN, phosphatase and tensin homolog; miR, microRNA; PI3K, phosphoinositide 3-kinase; -, phosphorylated; NC, negative control; LDH, lactate dehydrogenase; anti-371b-5p, anti-miR-371b-5p; PTEN I, PTEN inhibitor; OD, optical density.

expression, and induced AP-1 and KLF-2 protein expression, in an in vitro model of PAH, compared with the NC group. By contrast, miR-371b-5p transfection significantly induced eNOS, and suppressed AP-1 and KLF-2 protein expression levels, in an in vitro model of $\mathrm{PAH}$, compared with the $\mathrm{NC}$ group (Fig. 8E-H).

Inhibition of PTEN or PI3K affects miR-371b-5p-mediated effects on eNOS, AP-1 and KLF2 expression in an in vitro model of $P A H$. The present study investigated whether PTEN or PI3K inhibitor may regulate the effects of miR-371b-5p on downstream PTEN/PI3K/Akt signaling in an in vitro model of PAH. As presented in Fig. 9A-D, PTEN inhibitor significantly induced the expression of eNOS protein, and suppressed AP-1 and KLF-2 protein expression levels in PAECs following anti-miR-371b-5p transfection, compared with theanti-miR-371b-5p-transfected group without PTEN inhibitor treatment. By 
A

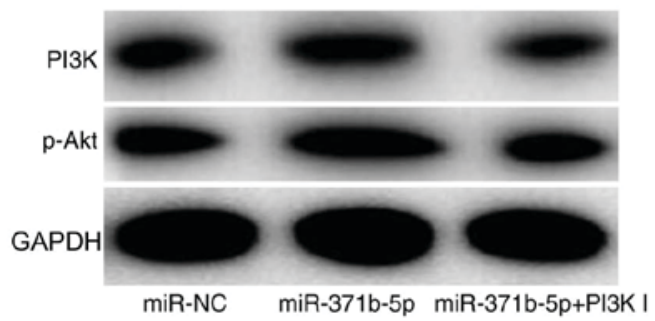

C

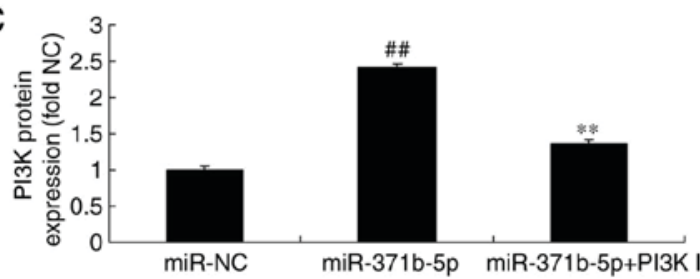

E

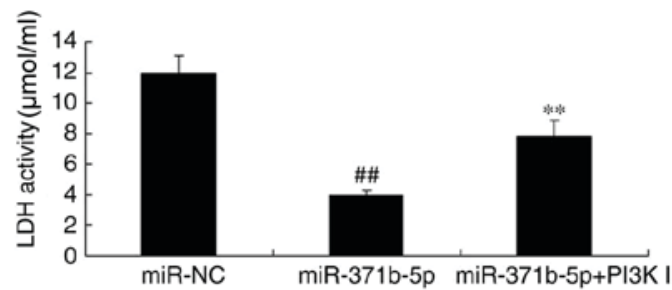

G

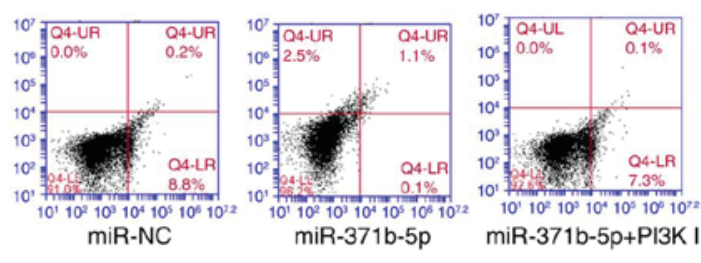

B

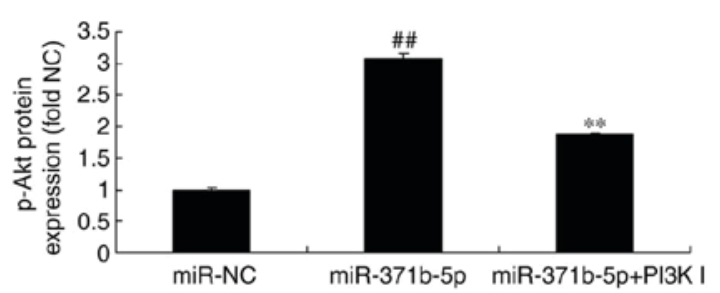

D

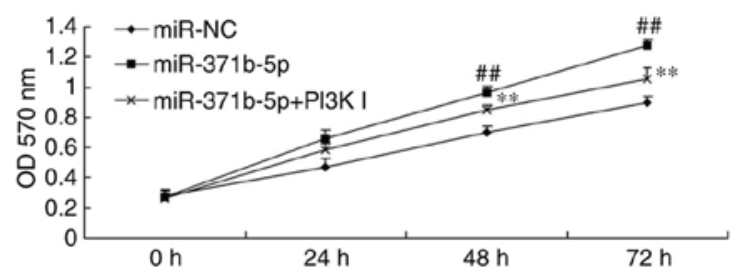

F

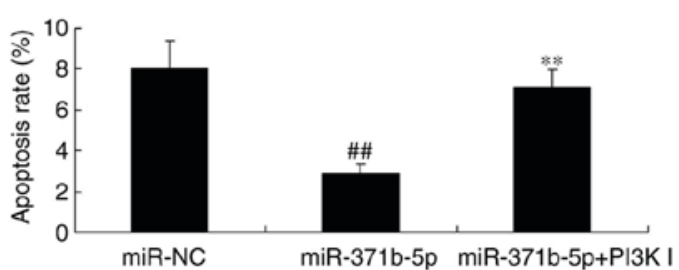

$\mathrm{H}$

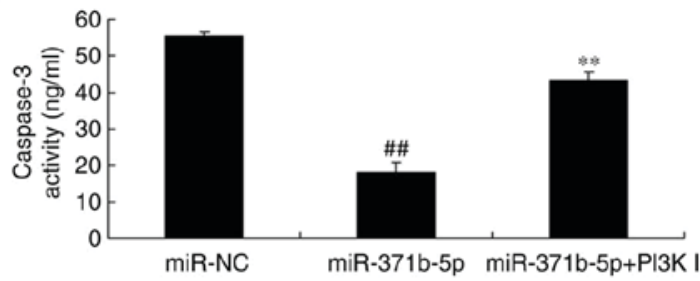

I

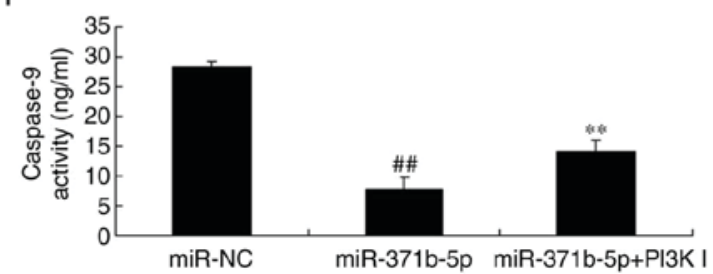

Figure 7. Inhibition of PI3K inhibits the effects of miR-371b-5p on pulmonary arterial endothelial cell death in rats with monocrotaline-induced pulmonary artery hypertension. (A) Representative western blot bands for the protein expression of PI3K and p-Akt following transfection of cells with miR-371b-5p, with or without PI3K inhibitor treatment, or miR-NC. Densitometric analysis was performed to quantify and statistically analyze the protein expression of (B) p-Akt and (C) PI3K. (D) Cell proliferation was measured by an MTT assay. (E) LDH activity was measured in cells transfected with miR-371b-5p, with or without PI3K inhibitor treatment, or miR-NC. (F) Cell apoptosis was quantified by flow cytometry following annexin V-fluorescein isothiocyanate/propidium iodide staining. (G) Q4-UR and Q4-LR indicate apoptotic cells). Representative flow cytometry plots following staining for apoptosis. The activity of (H) caspase-3 and (I) caspase-9 was also measured in cells transfected with miR-371b-5p, with or without PI3K inhibitor treatment, or miR-NC. ${ }^{\# \#<0.01 ~ v s . ~}$ miR-NC group; ${ }^{* *} \mathrm{P}<0.01$ vs. miR-371b-5p group. PI3K, phosphoinositide 3-kinase; miR, microRNA; p-, phosphorylated-; NC, negative control; LDH, lactate dehydrogenase; PI3K I, PI3K inhibitor; OD, optical density.

contrast, PI3K inhibitor significantly suppressed eNOS protein expression, and increased the expression of AP-1 and KLF-2 protein in PAECs following miR-371b-5p transfection, compared with the miR-371b-5p-transfected group without PI3K inhibitor treatment (Fig. 9E-H). These results demonstrated that miR-371b-5p upregulation may inhibit PAEC apoptosis in monocrotaline-induced PAH via PTEN/PI3K/Akt/eNOS-AP-1 and KLF-2 signaling pathways (Fig. 10).

\section{Discussion}

PAH is a clinical syndrome characterized by aprogressive increase in pulmonary vascular resistance (13). It induces pathological increases in PA pressure by blocking pulmonary circulation (13). Therefore, PAH can lead to right heart failure and mortality, and is associated with markedly poor prognosis (14). PAH has been regarded as the malignant tumor of the cardiovascular system (14). At present, anti-PAH drugs 
A

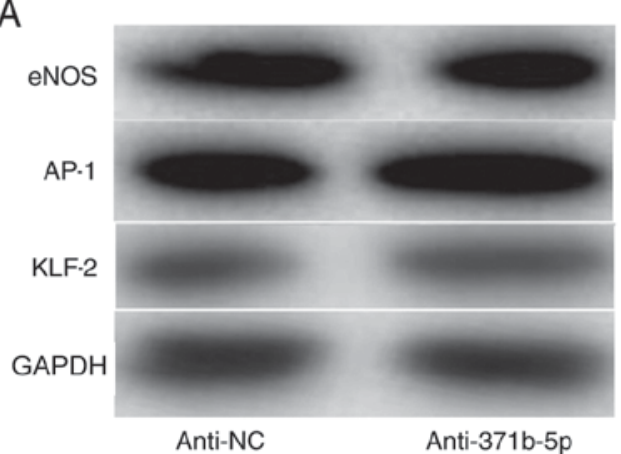

B

$\mathrm{C}$
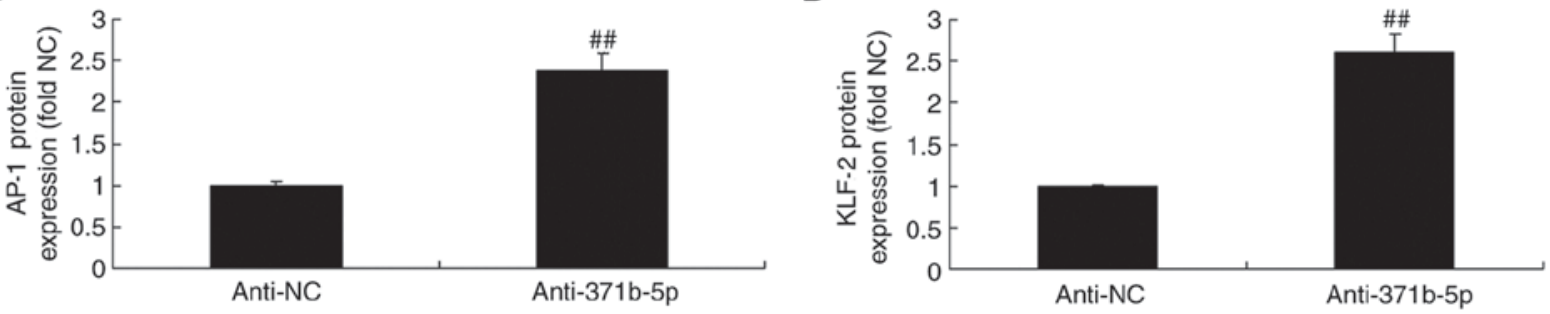

$\mathrm{E}$

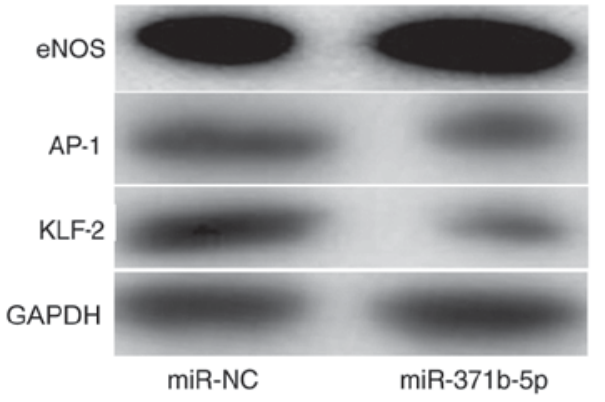

F

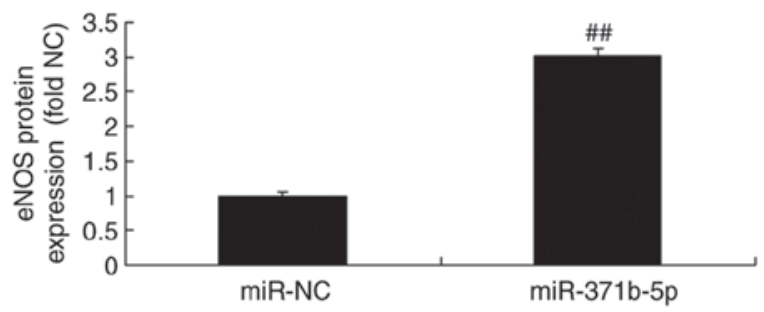

G

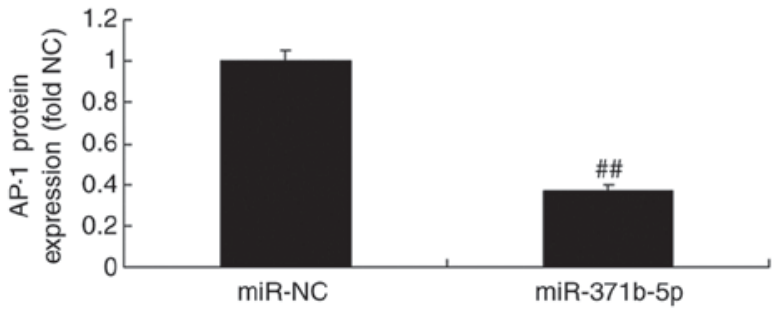

$\mathrm{H}$

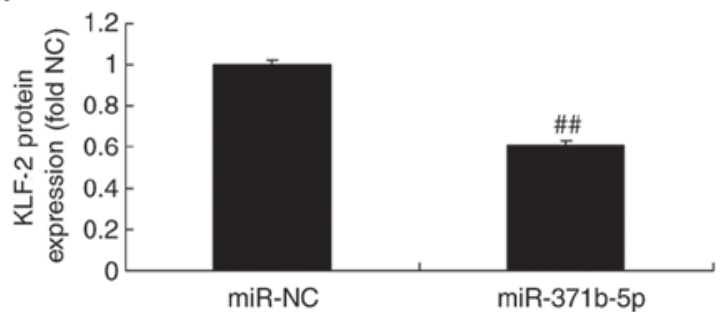

Figure 8. Effects of miR-371b-5p on eNOS, AP-1 and KLF-2 levels in pulmonary arterial endothelial cells from rats with monocrotaline-induced pulmonary arterial hypertension. (A) Representative western blot bands for the protein expression of eNOS, AP-1 and KLF-2 following transfection of cells with anti-miR-371b-5p or anti-NC. Densitometric analysis was performed to quantify and statistically analyze the protein expression of (B) eNOS, (C) AP-1 and (D) KLF-2. (E) Representative western blot bands for the protein expression of eNOS, AP-1 and KLF-2 following transfection of cells with miR-371b-5p or miR-NC. Densitometric analysis was performed to quantify and statistically analyze the protein expression of (F) eNOS, (G) AP-1 and (H) KLF-2."\#P<0.01 vs. anti-NC or miR-NC group. miR, microRNA; eNOS, endothelial nitric oxide synthase; AP-1, AP-1 transcription factor; NC, negative control; anti-371b-5p, anti-miR-371b-5p; KLF-2, Krüppel-like factor-2.

applied in the clinic are notably limited in China; such treatments are restricted to endothelial cell diastolic and systolic regulatory factors (14). Collectively, the findings of the present study indicated that miR-371b-5p may be downregulated in monocrotaline-induced PAH rat models, compared control rats.

miRNAs were first detected in the worm and fruit fly, and since then it is has been suggested in a study that miRNAs may be a key regulatory factor in the formation of mammalian cardiovascular disease (15). miRNAs regulate the expression of numerous mRNAs. Thus, miRNAs have important functions in biological processes (16). It has been reported that miRNAs have diverse functions in the cardiovascular system (15). The discovery of potential roles for miRNAs in the cardiovascular system has provided a novel area for research concerning the mechanism and prevention of cardiovascular disease (15). Furthermore, the present study revealed that an miR-371b-5p inhibitor increased cell apoptosis and reduced cell proliferation in monocrotaline-induced PAH cells.

A study concerning PTEN have been primarily focused in the cancer field (17). It has been demonstrated that a PTEN deletion or mutation may induce prostate cancer and breast 
A

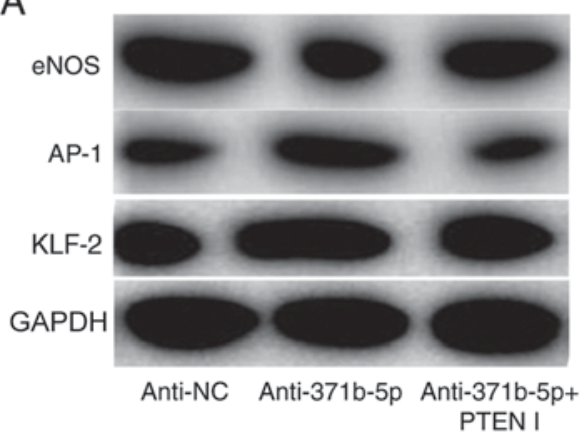

C

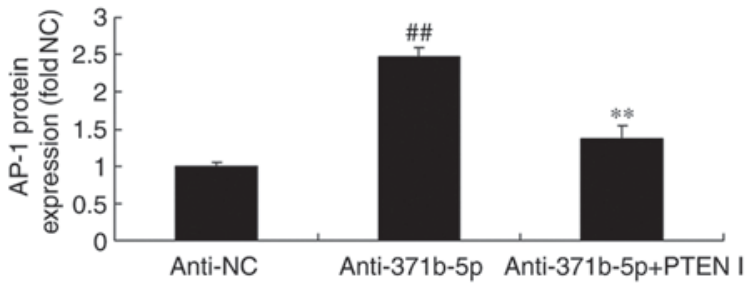

E

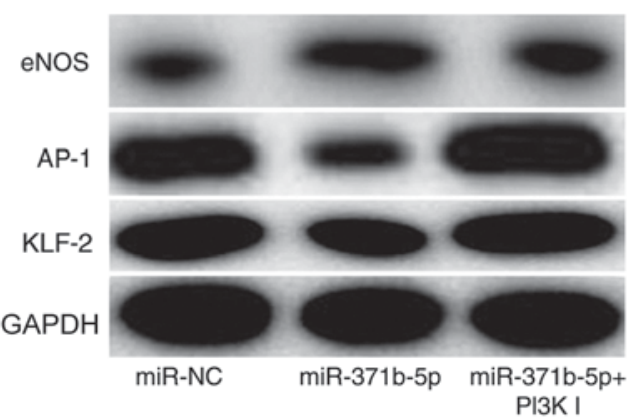

G

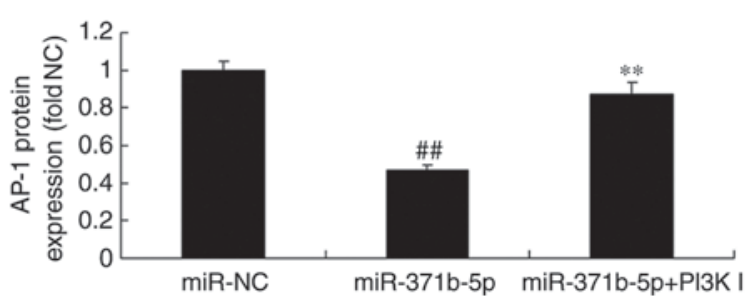

B

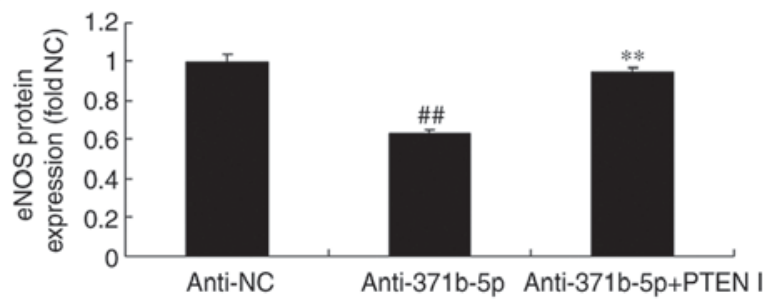

D

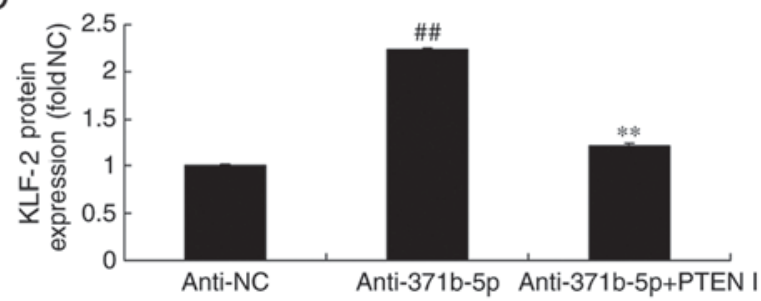

F

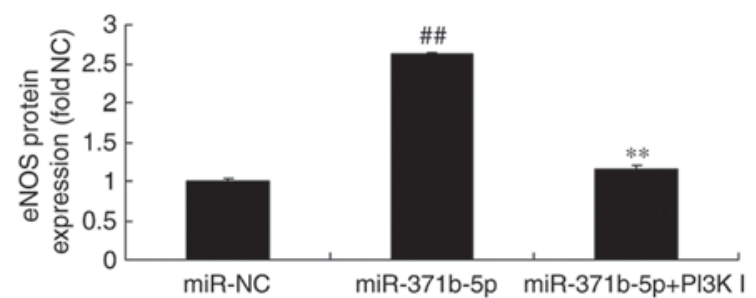

$\mathrm{H}$

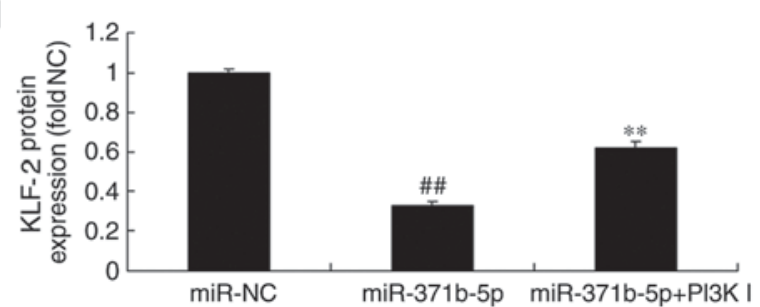

Figure 9. Inhibition of PTEN or PI3K affects miR-371b-5p-mediated effects on eNOS, AP-1 and KLF-2 levels in pulmonary arterial endothelial cells from rats with monocrotaline-induced pulmonary arterial hypertension. (A) Representative western blot bands for the protein expression of eNOS, AP-1 and KLF-2 following transfection of cells with anti-miR-371b-5p, with or without PTEN inhibitor treatment, or anti-NC. Densitometric analysis was performed to quantify and statistically analyze the protein expression of (B) eNOS, (C) AP-1and (D) KLF-2. (E) Representative western blot bands for the protein expression of eNOS, AP-1 and KLF-2 following transfection of cells with miR-371b-5p, with or without PI3K inhibitor treatment, or miR-NC. Densitometric analysis was performed to quantify and statistically analyze the protein expression of (F) eNOS, (G) AP-1 and (H) KLF-2. ${ }^{\# \#} \mathrm{P}<0.01$ vs. anti-NC or miR-NC group; ${ }^{* *} \mathrm{P}<0.01$ vs. anti-371b-5p or miR-371b-5p group. PTEN, phosphatase and tensin homolog; PI3K, phosphoinositide 3-kinase; miR, microRNA; eNOS, endothelial nitric oxide synthase; AP-1, AP-1 transcription factor; NC, negative control; anti-371b-5p, anti-miR-371b-5p; PTEN I, PTEN inhibitor; PI3K I, PI3K inhibitor; KLF-2, Krüppel-like factor-2.

cancer (17). In addition, it has previously been reported that PTEN inactivation in primary PA smooth muscle cells prompted smooth muscle cells to produce pro-inflammatory factors (18). Furthermore, inflammatory cell aggregation may lead to the development of PAH (19). PTEN expression has also been reported to promote the differentiation of smooth muscle progenitor cells to smooth muscle cells and their migration. Additionally, alterations in the PTEN/Akt signaling pathway have been associated with smooth muscle cell proliferation and intima formation $(7,18)$. Similarly, the present study reported that the inhibition of miR-371b-5p suppressed eNOS protein expression levels, and induced AP-1 and KLF-2 protein expression in PAECs from rats with monocrotaline-induced PAH via the PTEN/PI3K/Akt signaling pathway. Furthermore, Quan et al (20) revealed that the exosomal miR-371b-5p may promote proliferation by employing PTEN to orchestrate PI3K/Akt signaling within lung alveolar progenitor type II cells (20).

The PI3K/Akt signaling pathway is an important intracellular molecular signaling pathway and eNOS is a downstream target of the PI3K/Akt signaling pathway (21). NOS has three isomers (21); eNOS is the major pathway through which vascular endothelial cells produce NO (22). PI3K activates the phosphorylation of Akt and eNOS. In addition, it has been reported 


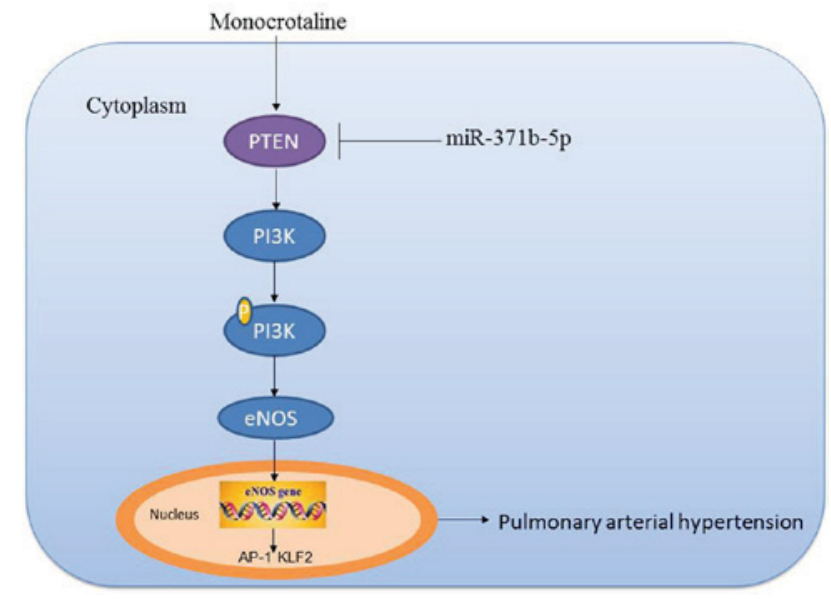

Figure 10. miR-371b-5p inhibits pulmonary arterial endothelial cell apoptosis in monocrotaline-induced pulmonary arterial hypertension via PTEN/PI3K/Akt signaling pathways. miR, microRNA; PTEN, phosphatase and tensin homolog; PI3K, phosphoinositide 3-kinase; eNOS, endothelial nitric oxide synthase; AP-1, AP-1 transcription factor; KLF-2, Krüppel-like factor-2.

to upregulate NO synthesis and secretion (22). Furthermore, the PI3K/Akt signaling pathway exerts protective effects on the cell proliferation of vascular endothelial cells and directly protect endothelial cell structure and function, thus potentially protecting the cardiovascular function (23). Estrogen has also been closely associated with eNOS and the promotion of NO synthesis and secretion. NO synthesis inhibits smooth muscle cell proliferation and reduces vascular tension (23). Collectively, the results of the present study indicated that treatment with a PTEN inhibitor inhibited the effects of anti-miR-371b-5p on cell apoptosis, while a PI3K inhibitor may mimic the effects of anti-miR-371b-5p on PAEC apoptosis.

In conclusion, the results of the present study demonstrated that miR-371b-5p upregulation may inhibit PAEC apoptosis in monocrotaline-induced PAH via the PTEN/PI3K/Akt/eNOS-AP-1 and KLF-2 signaling pathways, which may provide novel insight into the mechanisms of miR-371b-5p in the treatment of PAH.

\section{Acknowledgements}

Not applicable.

\section{Funding}

The present study was partly supported by a grant (grant no. 81241071) from the National Nature Science Foundation of China.

\section{Availability of data and materials}

The analyzed data sets generated during the study are available from the corresponding author on reasonable request.

\section{Authors' contributions}

GZ designed the experiment; WZ, YL and SW performed the experiment; GZ analyzed the data and wrote the manuscript.

\section{Ethics approval and consent to participate}

The present study was approved by the ethics committee of Beijing Anzhen Hospital (Beijing, China).

\section{Patient consent for publication}

Not applicable.

\section{Competing interests}

The authors declare that they have no competing interests.

\section{References}

1. Wu C, Guo J, Liu H, Pudasaini B, Yang W, Zhao Q, Wang L andeLiu J: The correlation of decreased heart rate recovery and chronotropic incompetence with exercise capacity in idiopathic pulmonary arterial hypertension patients. Biomed Res Int 2017: $3415401,2017$.

2. Galie N, Barbera JA, Frost AE, Ghofrani HA, Hoeper MM, McLaughlin VV, Peacock AJ, Simonneau G, Vachiery JL, Grünig E, et al: Initial use of ambrisentan plus tadalafil in pulmonary arterial hypertension. N Engl J Med 373: 834-844, 2015.

3. Li S, Ma Q, Yang Y, Lu J, Zhang Z, Jin M and Cheng W: Novel goal-directed hemodynamic optimization therapy based on major vasopressor during corrective cardiac surgery in patients with severe pulmonary arterial hypertension: A pilot study. Heart Surg Forum 19: E297-E302, 2016.

4. Dahan D, Hien TT, Tannenberg P, Ekman M, Rippe C, Boettger T, Braun T, Tran-Lundmark K, Tran PK, Swärd K and Albinsson S: MicroRNA-dependent control of serotonin-induced pulmonary arterial contraction. J Vasc Res 54: 246-256, 2017.

5. Xiao T, Xie L, Huang M and Shen J: Differential expression of microRNA in the lungs of rats with pulmonary arterial hypertension. Mol Med Rep 15: 591-596, 2017.

6. Nie X, Shi Y, Yu W, Xu J, Hu X and Du Y: Phosphorylation of PTEN increase in pathological right ventricular hypertrophy in rats with chronic hypoxia induced pulmonary hypertension. Chin Med J (Engl) 127: 338-342, 2014.

7. Liu Y, Cao Y, Sun S, Zhu J, Gao S, Pang J, Zhu D and Sun Z: Transforming growth factor-beta1 upregulation triggers pulmonary artery smooth muscle cell proliferation and apoptosis imbalance in rats with hypoxic pulmonary hypertension via the PTEN/AKT pathways. Int J Biochem Cell Biol 77: 141-154, 2016.

8. Ha D, Mester J, Eng C and Farha S: Pulmonary arterial hypertension in a patient with Cowden syndrome and the PTEN mutation. Pulm Circ 4: 728-731, 2014.

9. Yao L, Lu P, Li Y, Yang L, Feng H, Huang Y, Zhang D, Chen J and Zhu D: Osthole relaxes pulmonary arteries through endothelial phosphatidylinositol 3-kinase/Akt-eNOS-NO signaling pathway in rats. Eur J Pharmacol 699: 23-32, 2013.

10. Li L, Zhang X, Li X, Lv C, Yu H, Xu M, Zhang M, Fu Y, Meng H and Zhou J: TGF-beta1 inhibits the apoptosis of pulmonary arterial smooth muscle cells and contributes to pulmonary vascular medial thickening via the PI3K/Akt pathway. Mol Med Rep 13: 2751-2756, 2016.

11. Rabie T, Muhlhofer W, Bruckner T, Schwab A, Bauer AT, Zimmermann M, Bonke D, Marti HH and Schenkel J: Transient protective effect of B-vitamins in experimental epilepsy in the mouse brain. J Mol Neurosci 41: 74-79, 2010.

12. Livak KJ and Schmittgen TD: Analysis of relative gene expression data using real-time quantitative PCR and the 2(-Delta Delta C(T)) Method. Methods 25: 402-408, 2001.

13. Shea S, Lima J, Diez-Roux A, Jorgensen NW and McClelland RL: Socioeconomic status and poor health outcome at 10 years of follow-up in the multi-ethnic study of atherosclerosis. PLoS One 11: e0165651, 2016.

14. Saggar R, Khanna D, Vaidya A, Derhovanessian A, Maranian P, Duffy E, Belperio JA, Weigt SS, Dua S, Shapiro SS, et al: Changes in right heart haemodynamics and echocardiographic function in an advanced phenotype of pulmonary hypertension and right heart dysfunction associated with pulmonary fibrosis. Thorax 69: 123-129, 2014. 
15. Rothman AM, Arnold ND, Pickworth JA, Iremonger J, Ciuclan L, Allen RM, Guth-Gundel S, Southwood M, Morrell NW, Thomas M, et al: MicroRNA-140-5p and SMURF1 regulate pulmonary arterial hypertension. J Clin Invest 126: 2495-2508, 2016.

16. Sahoo S, Meijles DN, Al Ghouleh I, Tandon M, Cifuentes-Pagano E, Sembrat J, Rojas M, Goncharova E and Pagano PJ: MEF2C-MYOCD and leiomodin1 suppression by miRNA-214 promotes smooth muscle cell phenotype switching in pulmonary arterial hypertension. PLoS One 11: e0153780, 2016.

17. Zhao H, Lin G, Shi M, Gao J, Wang Y, Wang H, Sun H and Cao Y: The mechanism of neurogenic pulmonary edema in epilepsy. J Physiol Sci 64: 65-72, 2014.

18. Ravi Y, Selvendiran K, Meduru S, Citro L, Naidu S, Khan M, Rivera BK, Sai-Sudhakar CB and Kuppusamy P: Dysregulation of PTEN in cardiopulmonary vascular remodeling induced by pulmonary hypertension. Cell Biochem Biophys 67: 363-372, 2013.

19. Ravi Y, Selvendiran K, Naidu SK, Meduru S, Citro LA, Bognár B, Khan M, Kálai T, Hideg K, Kuppusamy P and Sai-Sudhakar CB Pulmonary hypertension secondary to left-heart failure involves peroxynitrite-induced downregulation of PTEN in the lung. Hypertension 61: 593-601, 2013.
20. Quan Y, Wang Z, Gong L, Peng X, Richard MA,Zhang J,Fornage M, Alcorn JL and Wang D: Exosome miR-371b-5p promotes proliferation of lung alveolar progenitor type II cells by using PTEN to orchestrate the PI3K/Akt signaling. Stem Cell Res Ther 8: 138, 2017.

21. Li L, Xu M, Li X, Lv C, Zhang X, Yu H, Zhang M, Fu Y, Meng H and Zhou J: Platelet-derived growth factor-B (PDGF-B) induced by hypoxia promotes the survival of pulmonary arterial endothelial cells through the PI3K/Akt/Stat3 pathway. Cell Physiol Biochem 35: 441-451, 2015.

22. Yuan P, Wu WH, Gao L, Zheng ZQ, Liu D, Mei HY, Zhang ZL and Jing ZC: Oestradiol ameliorates monocrotaline pulmonary hypertension via NO, prostacyclin and endothelin-1 pathways. Eur Respir J 41: 1116-1125, 2013.

23. Li H, Lu W, Cai WW, Wang PJ, Zhang N, Yu CP, Wang DL, Liu BC and Sun W: Telmisartan attenuates monocrotaline-induced pulmonary artery endothelial dysfunction through a PPAR gamma-dependent PI3K/Akt/eNOS pathway. Pulm Pharmacol Ther 28: 17-24, 2014.

(i) $\ominus$ This work is licensed under a Creative Commons Attribution-NonCommercial-NoDerivatives 4.0 International (CC BY-NC-ND 4.0) License. 